\title{
Reactions with Exotic Nuclei at Near and Sub-barrier Energies
}

\author{
Lei Yang ${ }^{1}$, Chengjian Lin ${ }^{1,2, *}$, Huiming $\mathrm{Jia}^{1}$, Nanru $\mathrm{Ma}^{1 * *}$, Dongxi Wang ${ }^{1}$, Feng Yang ${ }^{1}$, Lijie Sun ${ }^{1 * * *}$, Xinxing Xu $^{1}$, \\ Peiwei Wen ${ }^{1 * * *}$, and Fupeng Zhong ${ }^{1,2}$ \\ ${ }^{1}$ China Institute of Atomic Energy, P. O. Box 275(10), Beijing 102413, China \\ ${ }^{2}$ Department of Physics, Guangxi Normal University, Guilin 541004, China
}

\begin{abstract}
We will introduce some new results derived recently by the nuclear reaction group at China Institute of Atomic Energy, on the properties of the optical model potentials of neutron-halo ${ }^{6} \mathrm{He}$ system and reaction mechanisms induced by proton-drip line nucleus ${ }^{17} \mathrm{~F}$ at energies around the Coulomb barrier. For the study of optical model potentials of exotic nuclear system, we proposed a novel method, i.e., the transfer reaction method. This method has been applied to extract the optical potentials of neutron-halo ${ }^{6} \mathrm{He}+{ }^{209} \mathrm{Bi}$ system by measuring the one-proton transfer reactions induced by ${ }^{7} \mathrm{Li}$ on a ${ }^{208} \mathrm{~Pb}$ target. A complete picture of threshold anomaly behavior was obtained in the ${ }^{6} \mathrm{He}+{ }^{209} \mathrm{Bi}$ system for the first time, where a decreasing trend of the depth of the imaginary potential is observed in the deep sub-barrier region, and the reaction threshold energy is extracted. Moreover, results show that the dispersion relation is not applicable for this exotic nuclear system. Reaction mechanisms of proton-rich nuclear systems ${ }^{17} \mathrm{~F}+{ }^{89} \mathrm{Y}$ and ${ }^{58} \mathrm{Ni}$ were also studied at energies around the Coulomb barrier. Continuum discretized coupled-channels calculations indicate that the coupling effects of the continuum states of ${ }^{17} \mathrm{~F}+{ }^{89} \mathrm{Y}$ is not significant. Thanks to the employment of a powerful ionization-chamber based detector array, the reaction products over a large $Z$ in the systems of ${ }^{17} \mathrm{~F}+{ }^{58} \mathrm{Ni}$ can be identified clearly. The data analysis is undergoing.
\end{abstract}

\section{Introduction}

The availability of relatively intense Radioactive Ion Beams (RIBs) has caused a revival of interest in the nuclear physics. For nuclear reactions at near- and sub-barrer energies, some surprising results in the elastic scattering, direct and fusion reactions induced by RIBs have been reported $[1,2]$. These nuclei are often labelled as "exotic" because they have unusual properties, e.g., small separation energies for the valence particles, extensive matter distribution, exhibiting the halo/skin or cluster phenomena and so on. The nuclear interaction is a fundamental ingredient in the study of mechanisms of nuclear reactions. The optical model potential (OMP) is generally adopted to phenomenologically describe the interaction of nuclear collisions. It is well known that the OMPs are closely related to the internal structures of the colliding nuclei, thus the OMPs of exotic nuclear systems are expected to present unusual behaviors. In this paper, we will introduce some new results obtained by the nuclear reaction group at CIAE (China Institute of Atomic Energy) recently, on the properties of the OMPs of neutron-halo ${ }^{6} \mathrm{He}$ systems and the reaction mechanisms induced by the proton-drip line nucleus ${ }^{17} \mathrm{~F}$ at energies around the Coulomb barrier.

\footnotetext{
*Corresponding author: e-mail: cjlin@ ciae.ac.cn

** Present address: Center for Nuclear Study, University of Tokyo

*** Present address: Michigan State University

**** Present address: Joint Institute for Nuclear Research, Dubna
}

\section{OMPs of neutron-halo ${ }^{6} \mathrm{He}$ systems}

The OMP can be expressed as $U(r)=V(r)+i W(r)$, where the real part describes the elastic scattering process while the imaginary part represents the absorption of incident flux, i.e., all the nonelastic reaction processes. The properties of the OMPs of tightly bound systems have been recognized with decades of research, e.g., when the interaction energy gets close to the Coulomb barrier, a strong energy dependence is present in both the real and the imaginary parts, which is known as the threshold anomaly [35]. This behavior is characterized by a sharp decrease in the imaginary part as the bombarding energy decreases towards the Coulomb barrier, associated with a localized bell-shaped structure around the barrier in the real part. The dispersion relation can be used to connect the real and imaginary parts [6]. The dispersion relation is a natural consequence of the causality principle, describing the effect of dispersion in a medium on the properties of a wave traveling within that medium.

It is well known that the OMPs are closely related to the internal structures of the colliding nuclei, thus particular properties of the potential arising from the exotic nuclear structure can be expected [7,8]. Conventionally, OMPs are extracted by fitting angular distributions of elastic scattering. At energies close to and below the barrier, however, the angular distributions become insensitive to the nuclear interaction potential. It becomes much more worse for the reactions induced by unstable nuclei, because of limitations of the intensity and/or the phase-space 
qualities of RIBs. In view of this fact, we proposed a novel method, i.e., the transfer reaction method [9], to study the OMPs of exotic systems by the utilization of a stable beam. The detail of the transfer reaction method can be found in Refs. [10, 11], where the sensitivity test and the comparison with the traditional elastic scattering method were also introduced.

We have applied the transfer reaction method to study the OMPs of neutron-halo ${ }^{6} \mathrm{He}$ systems, by measuring the one-proton transfer reactions induced by ${ }^{7} \mathrm{Li}$ beam accelerated by the HI-13 tandem accelerator at CIAE. Three systems have been measured at energies around the Coulomb barrier, i.e., ${ }^{11} \mathrm{~B}\left({ }^{7} \mathrm{Li},{ }^{6} \mathrm{He}\right){ }^{12} \mathrm{C},{ }^{63} \mathrm{Cu}\left({ }^{7} \mathrm{Li},{ }^{6} \mathrm{He}\right){ }^{64} \mathrm{Zn} \mathrm{[12]}$ and ${ }^{208} \mathrm{~Pb}\left({ }^{7} \mathrm{Li},{ }^{6} \mathrm{He}\right){ }^{209} \mathrm{Bi}[11,13,14]$, to study the OMPs of the light system ${ }^{6} \mathrm{He}+{ }^{12} \mathrm{C}$, the medium-mass system ${ }^{6} \mathrm{He}+{ }^{64} \mathrm{Zn}$ and the heavy system ${ }^{6} \mathrm{He}+{ }^{209} \mathrm{Bi}$. Because the lowest terminal high voltage of the tandem accelerator is limited to $3 \mathrm{MV}$, corresponding to about $12 \mathrm{MeV}$ of ${ }^{7} \mathrm{Li}$ beam energy. Therefore, the deep sub-barrier region can be achieved only for the ${ }^{6} \mathrm{He}+{ }^{209} \mathrm{Bi}$ system. The angular distributions of the transfer reaction ${ }^{208} \mathrm{~Pb}\left({ }^{7} \mathrm{Li},{ }^{6} \mathrm{He}\right){ }^{209} \mathrm{Bi}$ at the lowest two incident energies (21.20 and $24.30 \mathrm{MeV}$ ) are shown in Fig. 1. The corresponding elastic scattering data of ${ }^{7} \mathrm{Li}+{ }^{208} \mathrm{~Pb}$ at $E_{\mathrm{lab}}\left({ }^{7} \mathrm{Li}\right)=24.30 \mathrm{MeV}$ and ${ }^{6} \mathrm{He}+{ }^{209} \mathrm{Bi}$ at $E_{\text {lab }}\left({ }^{6} \mathrm{He}\right)=17.84 \mathrm{MeV}$ [15] are also shown in Fig. 1 (b). The transfer reaction data were fitted by the coupled-reaction-channels (CRC) approach, to extract the OMPs of ${ }^{6} \mathrm{He}+{ }^{209} \mathrm{He}$. The fitting results and that of the optical model calculations with the OMPs extracted for ${ }^{6} \mathrm{He}+{ }^{209} \mathrm{Bi}$ are also present in Fig. 1.

The energy dependence of the real and imaginary potentials of ${ }^{6} \mathrm{He}+{ }^{209} \mathrm{Bi}$ at the sensitivity radius of $13.5 \mathrm{fm}$ is shown in Fig. 2. As shown in Fig. 2(b), the depth of the imaginary potential decreased as the energy reduced in the deep sub-barrier region, which vanishes at $13.73 \pm 1.63 \mathrm{MeV}$ (corresponding to $0.68 V_{\mathrm{B}}$ ) according to the extrapolation, where the threshold energy emerges. It is the first time that the reaction threshold is observed for a halo nuclear system. Compared with the threshold values of $0.93 V_{\mathrm{B}}$ in the tightly bound ${ }^{16} \mathrm{O}+{ }^{208} \mathrm{~Pb}$ system [4] and $0.81 V_{\mathrm{B}}$ in the weakly bound ${ }^{6} \mathrm{Li}+{ }^{144} \mathrm{Sm}$ system [16], such a low threshold energy indicates the feature of the extended matter distribution of the halo nucleus ${ }^{6} \mathrm{He}$. Furthermore, according to the linear segment fitting of the imaginary potential as shown in Fig. 2(b), the dispersion relation calculation result with the linear schematic model [6] for the real part is shown in Fig. 2(a). It can be seen clearly the prediction of the dispersion relation cannot reproduce the experimental results. The possible reasons for the abnormal behavior are listed as follows [11, 14]: (a) the dispersion relation is deduced from the KramersKronig relation, which is derived from Cauchy residue theorem. One prerequisite of the theorem is that there should be a finite list of isolated singularities, corresponding to the discrete states of the interacting nuclear system [6]. For the weakly bound system, however, the continuum states may play a more important role. Therefore, it is not mathematically rigorous to apply the dispersion relation directly to the exotic nuclear system; (b) the OMPs extracted from experimental data is a equivalent local poten-

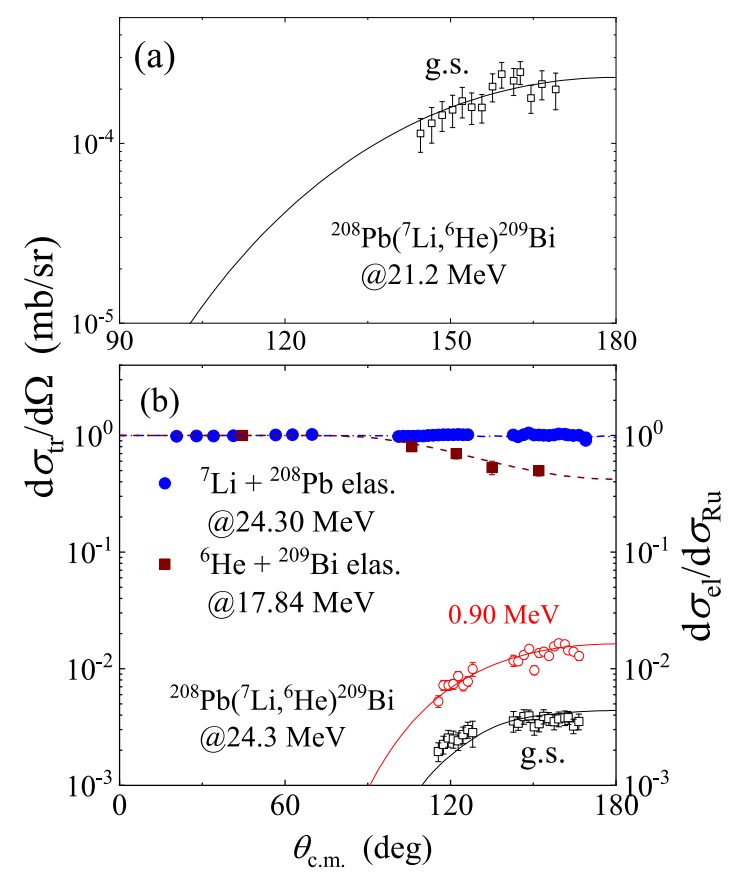

Figure 1. (Color online) Angular distributions of ${ }^{208} \mathrm{~Pb}\left({ }^{7} \mathrm{Li},{ }^{6} \mathrm{He}\right)$ reactions for proton transferred to different states of ${ }^{209} \mathrm{Bi}$ at $E_{\text {lab }}=21.20$ (a) and 24.30 (b) $\mathrm{MeV}$, respectively. The experimental results are labelled according to the the excitation energy of ${ }^{209} \mathrm{Bi}$. The solid curves represent the CRC fitting results. The elastic scattering of the entrance channel ${ }^{7} \mathrm{Li}+{ }^{208} \mathrm{~Pb} E_{\text {lab }}\left({ }^{7} \mathrm{Li}\right)=$ $24.30 \mathrm{MeV}$ as well as that of the corresponding exit channel ${ }^{6} \mathrm{He}$ $+{ }^{209} \mathrm{Bi}$ at $E_{\text {lab }}=17.84 \mathrm{MeV}$ [15] are also shown by the solid circles and squares, respectively. The dash-dotted curve is the CRC fitting result and the dashed curve represents the calculation result with the OMP extracted from the transfer reaction.

tial, which will introduce the so-called "spurious" energy dependence. Such a "spurious" dependency is not required to follow the dispersion relation; (c) the derived OMPs are only sensitive to the region located in the external part of the potential. However, the dispersion relation is derived only for a potential that can yield the wave function over all spatial regions. Thus the phenomenological potential, which can only generate the wave function in the external region, does not necessarily need to abide by the causality property [17]. Further investigation is strongly required to discover the underlying physics.

\section{Reaction mechanisms of ${ }^{17} \mathrm{~F}$ systems}

Based on the understanding of the OMPs of weakly bound nuclear systems, we can move a further step to study the reaction mechanisms induced by exotic nuclei. There is already systematic knowledge of the reaction mechanisms of neutron-halo nuclear systems [1], for example, the neutron transfer reaction is dominant for the direct reactions; and for the fusion reaction, the fusion cross section is suppressed in the above barrier region, but enhanced below the barrier. For the proton-rich side, however, the experimental is still scarce. Therefore, we performed a series of experiments to study the reactions induced by the protondrip line nucleus ${ }^{17} \mathrm{~F}$ at energies around the Coulomb bar- 


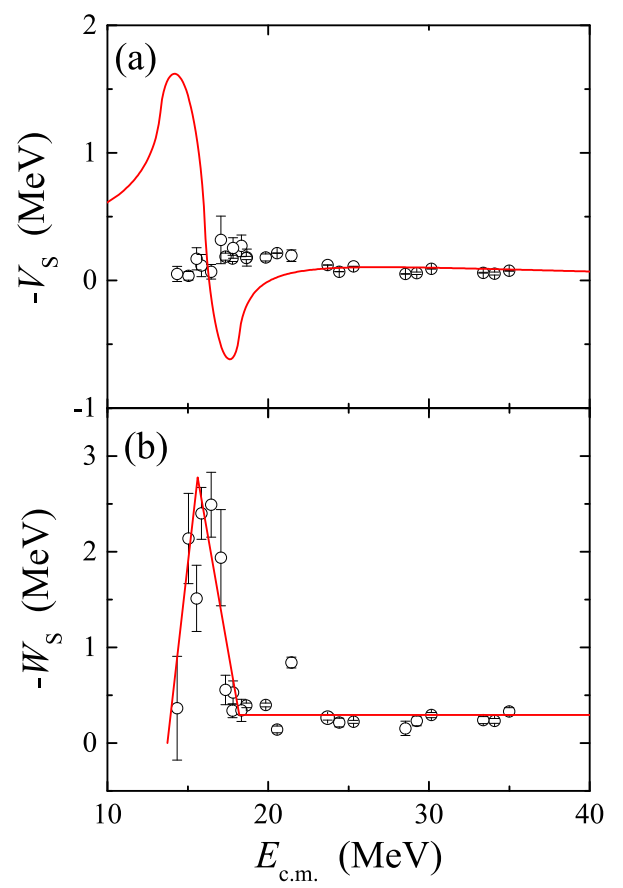

Figure 2. Energy dependence of the depths of real (a) and imaginary (b) potentials at the sensitivity radius of $13.5 \mathrm{fm}$ for the ${ }^{6} \mathrm{He}$ $+{ }^{209} \mathrm{Bi}$ system $[11,13,14]$. Curves in (b) represent the linear segment fitting for the imaginary potential. The predictions of the dispersion relation according to the variations of the imaginary potentials are present in (a).

rier, such as ${ }^{17} \mathrm{~F}+{ }^{89} \mathrm{Y}[18],{ }^{17} \mathrm{~F}+{ }^{208} \mathrm{~Pb}[19]$ at the Radioactive Ion Beam Line at the Heavy Ion Research Facility in Lanzhou (HIRFL-RIBLL1) [20] and ${ }^{17} \mathrm{~F}+{ }^{58} \mathrm{Ni}$ [21] at the low energy radioactive isotope beam separator CRIB (Center for Nuclear Study Radioactive Ion Beam separator) of University of Tokyo [22]. The valence proton of ${ }^{17} \mathrm{~F}$ is bound only by $0.6 \mathrm{MeV}$. The root mean square $(\mathrm{rms})$ radius of the ground state is $3.7 \mathrm{fm}$ [23], which is significantly larger than that of the ${ }^{16} \mathrm{O}$ core. The first excited state of ${ }^{17} \mathrm{~F}, E_{x}=0.495 \mathrm{MeV}$ and $J^{\pi}=1 / 2^{+}$, is the only bound state below the breakup threshold, and reported to have an extended rms radius, $5.3 \mathrm{fm}$ [23], exhibiting a proton-halo structure [24].

The elastic scattering of ${ }^{17} \mathrm{~F}+{ }^{89} \mathrm{Y}$ was measured at $E_{\text {lab }}\left({ }^{17} \mathrm{~F}\right)=50.0$ and $59.0 \mathrm{MeV}$ [18]. Continuum discretized coupled-channels (CDCC) and one-channel calculations were performed to understand breakup coupling effects on the elastic scattering. The experimental and theoretical results are shown in Fig.3. According to the comparison, both the CDCC and one-channel calculations can reproduce the elastic scattering data reasonably well, indicating that the effect of the coupling to continuum states (both the bound-continuum states and the continuum-continuum state couplings) is not significant.

For the measurements of ${ }^{17} \mathrm{~F}+{ }^{58} \mathrm{Ni}$ and ${ }^{208} \mathrm{~Pb}$, an innovatively designed Multilayer Ionization-chamber (IC) Telescope Array (MITA) [21] was employed to detect the reaction products over a large range of $Z$. Thanks to the powerful array, both the light reaction products like $p$ and

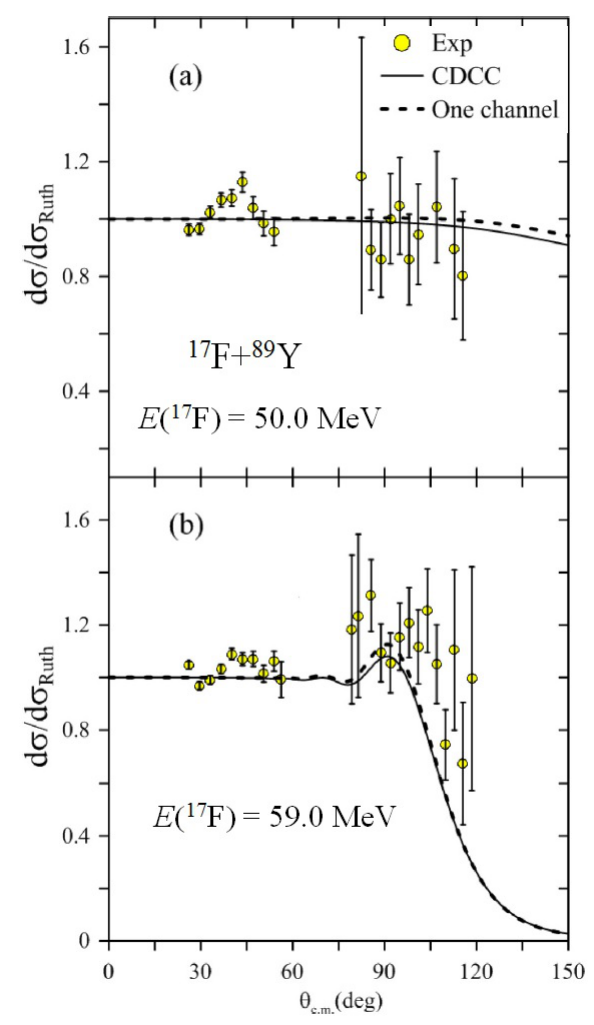

Figure 3. Angular distributions of elastic scattering of ${ }^{17} \mathrm{~F}$ on ${ }^{89} \mathrm{Y}$ at $E_{\mathrm{lab}}\left({ }^{17} \mathrm{~F}\right)=50.0$ (a) and 59.0 (b) $\mathrm{MeV}$ [18]. The solid and dashed curves represent the CDCC and one-channel calculations.

$\alpha$, and the heavy ions like ${ }^{16} \mathrm{O}$ and ${ }^{17} \mathrm{~F}$, can be clearly distinguished. The angular distributions of elastic scattering of ${ }^{17} \mathrm{~F}+{ }^{58} \mathrm{Ni}$ are shown in Fig. 4, where the CDCC calculation results are also presented. The data analysis of these two systems is still in progress.

\section{Summary}

With the application of the transfer reaction method, the OMPs of neutron-halo ${ }^{6} \mathrm{He}+{ }^{209} \mathrm{Bi}$ system were extracted exactly. In the sub-barrier energy region, the depth of the imaginary potential increases first with energy decreasing below the barrier and then falls quickly down to 0 . According to the extrapolation, the reaction threshold energy is determined for the first time for a halo nuclear system. Moreover, the dispersion relation cannot describe the connection between the real and imaginary potentials. Some possible reasons are discussed for such a peculiar behavior, but further study is still desired for the underlying physics.

We also performed experiments to study the reactions induced by the proton-rich nucleus ${ }^{17} \mathrm{~F}$. Three systems, i.e., ${ }^{17} \mathrm{~F}+{ }^{58} \mathrm{Ni},{ }^{89} \mathrm{Y}$ and ${ }^{208} \mathrm{~Pb}$, were measured at energies around the Coulomb barrier. For the ${ }^{17} \mathrm{~F}+{ }^{89} \mathrm{Y}$ system, only the elastic scattering data were derived due to the limited particle-identification capability of the silicon detector telescope. According to the CDCC and one-channel calculations, the breakup coupling effects on the elastic scattering of ${ }^{17} \mathrm{~F}+{ }^{89} \mathrm{Y}$ is insignificant. For the measurement of 


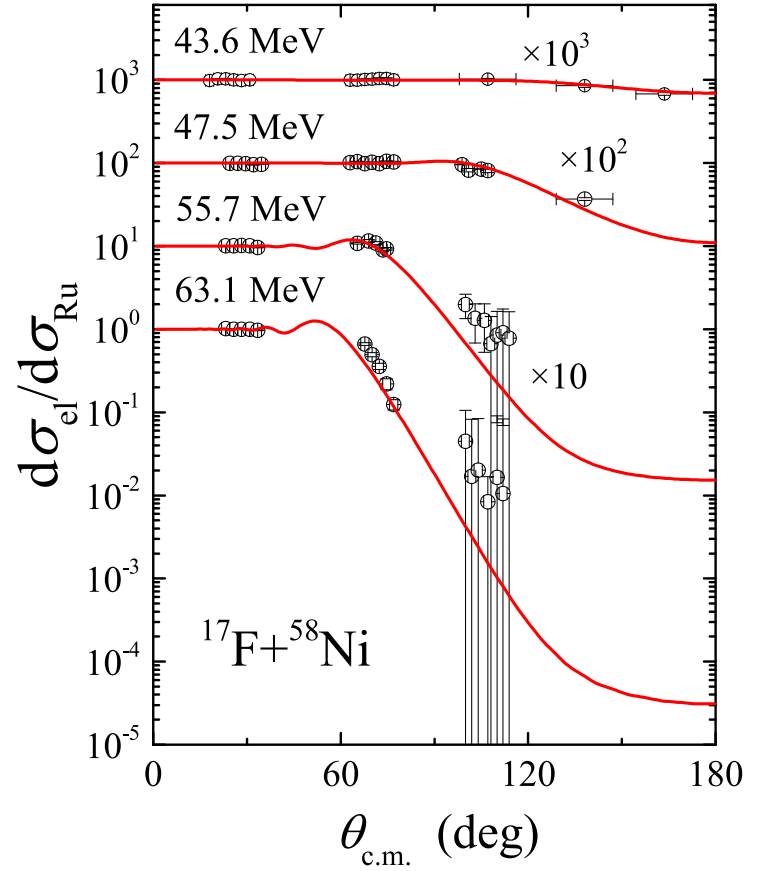

Figure 4. Angular distributions of elastic scattering of ${ }^{17} \mathrm{~F}$ on ${ }^{58} \mathrm{Ni}$ at $E_{\mathrm{lab}}\left({ }^{17} \mathrm{~F}\right)=43.6,47.5,55.7$ and $63.1 \mathrm{MeV}$. The solid curves represent the CDCC calculations.

${ }^{17} \mathrm{~F}+{ }^{89} \mathrm{Y}$ and ${ }^{208} \mathrm{~Pb}$, a ionization chamber based detector array was employed, to identify the reaction products over a large $Z$ region. The data analysis is still undergoing.

\section{Acknowledgements}

We acknowledge the related physicists and operations staff of the HI-13 tandem accelerator, the HIRFL-RIBLL1, and the CNS-CIRB for providing good quality beams and performing the experiments. This work is supported by the National Key R\&D Program of China (Contract No. 2018YFA0404404), the National Natural Science Foundation of China (Grants Nos.11635015, U1732145, 11705285, U1867212, 11805280, and 11811530071), and the Project funded by China Postdoctoral Science Foundation (Grant No. 2017M621035).

\section{References}

[1] L. F. Canto, P. R. S. Gomes, R. Donangelo et al., Phys. Rep. 596, 1 (2015).
[2] J. J. Kolata, V. Guimarães, and E. F. Aguilera, Eur. Phys. J. A 52, 123 (2016).

[3] B. R. Fulton, D. W. Banes, J. S. Lilley et al., Phys. Lett. B 162, 55 (1985).

[4] M. A. Nagarajan, C. C. Mahaux, G. R. Satchler, Phys. Rev. Lett. 54, 1136 (1985).

[5] C. J. Lin, J. C. Xu, H. Q. Zhang et al., Phys. Rev. C 63, 064606 (2001).

[6] C. Mahaux, H. Ngô, G. R. Satchler, Nucl. Phys. A 449, 354 (1986).

[7] A. M. Sánchez-Benítez, D. Escrig, M. A. G. Álvarez et al., Nucl. Phys. A 803, 30 (2008).

[8] M. S. Hussein, P. R. S. Gomes, J. Lubian and L. C. Chamon, Phys. Rev. C 73, 044610 (2006).

[9] C. J. Lin, F. Yang, P. Zhou et al., AIP Conf. Proc. 853, 81 (2006).

[10] Z. D. Wu, L. Yang, C. J. Lin et al., Chin. Phys. Lett. 31, 092401 (2014).

[11] L. Yang, C. J. Lin, H. M. Jia et al., Phys. Rev. C 96, 044615 (2017).

[12] L. Yang, C. J. Lin, H. M. Jia et al., Phys. Rev. C 95, 034616 (2017).

[13] L. Yang, C. J. Lin, H. M. Jia et al., Phys. Rev. C 89, 044615 (2014).

[14] L. Yang, C. J. Lin, H. M. Jia et al., Phys. Rev. Lett. 119, 042503 (2017).

[15] E. F. Aguilera, J. J. Kolata, F. D. Becchetti et al., Phys. Rev. C 63, 061603(R) (2001).

[16] J. M. Figueira, J. O. Fernández Niello, A. Arazi et al., Phys. Rev. C 81, 024613 (2010).

[17] R. Lipperheide and A. K. Schmidt, Nucl. Phys. A 112, 65 (1968).

[18] G. L. Zhang, G. X. Zhang, C. J. Lin et al., Phys. Rev. C 97, 044618 (2018).

[19] Nan-Ru Ma, Cheng-Jian Lin, Jian-Song Wang et al., Chin. Phys. C 40, 116004 (2016).

[20] Z. Sun et al., Nucl. Instrum. Methods Phys. Res. A 503, 496 (2003).

[21] N. R. Ma, L. Yang, C. J. Lin et al., Eur. Phys. J. A 55, 87 (2019).

[22] S. Kubono et al., Eur. Phys. J. A 13, 217 (2002).

[23] R. Morlock, R. Kunz, A. Mayer et al., Phys. Rev. Lett. 79, 3837 (1997).

[24] R. Lewis and A. C. Hayes, Phys. Rev. Lett. 59, 1211 (1999). 